\title{
The Role of Pharmacovigilance and ISoP During the Global COVID-19 Pandemic
}

\author{
Rebecca E Chandler ${ }^{1}$ (1) $\cdot$ Deirdre McCarthy ${ }^{2} \cdot$ Jean-Christophe Delumeau ${ }^{3} \cdot$ Mira Harrison-Woolrych $^{4}$
}

Published online: 7 May 2020

(c) The Author(s) 2020

Today we find ourselves living the pages of the history books of the next generation. The global pandemic, long predicted by experts in the fields of infectious diseases and global public health, has arrived and brought the life to which we had grown so accustomed to an abrupt halt. However, our work must continue as the need for expertise in medicines safety has been evident from the first days of the pandemic. What is the role of pharmacovigilance in the pandemic situation? More specifically, what is the role of the International Society of Pharmacovigilance (ISoP) during the current coronavirus 2019 (COVID-19) outbreak?

Monitoring the safety of medicines is an obvious top priority. A collection of therapies for consideration in the treatment of coronavirus infections has been identified and is currently being deployed in many countries. Within the World Health Organization (WHO)-led global Solidarity trial, the use of new drugs, such as remdesivir, and of 'repurposed' drugs, such as hydroxychloroquine and lopinavir/ritonavir, are being explored for effectiveness against COVID-19 [1]. Multiple vaccines of different types are under development [2], and the first participants in clinical trials have received the first injections. Reports of suspected adverse drug reactions for these different therapies have already reached Vigibase, the global database of individual case safety reports, managed by the Uppsala Monitoring Centre (personal knowledge). A true opportunity to bring

Rebecca Chandler, Deirdre McCarthy, Jean-Christophe Delumeau and Mira Harrison-Woolrych: Members of the Executive Committee of ISoP.

\section{Rebecca E Chandler}

rebecca.chandler@who-umc.org

1 Uppsala Monitoring Centre, Uppsala, Sweden

2 IQVIA, Cambridge, MA, USA

3 Bayer (South East Asia) Pte Ltd., Singapore, Singapore

4 International Society of Pharmacovigilance (ISoP), London, UK pharmacovigilance closer to healthcare systems arises; clinicians, faced with treatment decisions in the absence of data from randomised controlled trials, will use available real-life observational data on both treatment benefits and harms. Integration of pharmacovigilance with clinical practice is a key area of interest to many ISoP members, often discussed within our chapters and special interest groups (SIGs) and at our meetings around the world. Timely review of incoming data and real-time signal detection can provide important safety information for healthcare providers. Therefore, ensuring and supporting the collection of high-quality data from adverse drug reaction reports in all countries will be essential responsibilities of our daily work.

Risk communication, difficult under normal circumstances, will be an even greater challenge given the amount of uncertainty that surrounds all aspects of the pandemic. The ISoP risk communication SIG and the ISoP communications team have been discussing the role of our professional society in relaying information about the COVID-19 pandemic, particularly regarding the efficacy and safety of medicines and vaccines for the treatment and prevention of this novel virus. Some ISoP initiatives under development are summarised near the end of this article. Attention will also be required to detect false information, and we will endeavour to provide explanations and corrections in an effort to prevent harm.

The collective lack of experience worldwide in managing responses from a public health perspective has been made apparent by the differences in approaches between countries to containment/mitigation strategies and regulatory recommendations for treatment options. In our hyper-connected world with its 24-h news coverage, the public is aware of the lack of agreement between different governmental authorities and between experts within individual countries, making the need for transparency and honesty in the communication of 'uncertainties' imperative.

Sharing and collaboration, both within and beyond our pharmacovigilance community, will be integral. During the 
emergence of the COVID-19 pandemic, the ISoP executive committee (EC) has been directly in touch with individual ISoP members around the world. We know that many colleagues are working at the front line of clinical care or are in close support of others in key roles, and we want to offer the assistance of ISoP to any of you who need it, in whatever way we can help. A great strength of ISoP is the professional and personal support we provide to each other in the work we are performing around the world. Support of our pharmacovigilance colleagues in lower-income countries may be necessary as they will need to manage the same challenges with far more limited resources. Sharing of knowledge and insight into regulatory decisions as well as communication materials/social media campaigns can help to unload the burden of the few people often employed in many smaller national pharmacovigilance centres. Collaboration with other scientific organisations can demonstrate of the value of spontaneous reports as a form of real-world data. Queries of spontaneous databases combined with real-time observational studies using data from big data networks of insurance claims and electronic healthcare records can allow for more efficient evidence generation to explore any medicines safety issues that arise.

In view of the current situation, the ISoP EC has made some important changes to our strategic plan for 2020. Most of our face-to-face conferences and meetings this year, including our annual meeting which this year was planned to take place in Oman, will be rescheduled to 2021. Planning will continue to take place during 2020 for these meetings, and we look forward to providing you with updates in due course (please check the ISoP website at https://isoponline .org/).

In addition to re-planning the above events, we are continuing other important ISoP work during 2020, including the following key initiatives:

1. Monitoring relevant publications on medicines and vaccines used to treat/prevent COVID-19.

2. Supporting regional pharmacovigilance societies during the COVID-19 pandemic.

3. Developing ISoP infographics to communicate pharmacovigilance advice to patients and healthcare professionals worldwide.
4. Conducting ISoP webinars in collaboration with Drug Safety, available at https://isoponline.org/members/ webinars/.

5. Encouraging ISoP chapters and SIGs to continue relevant work and share key outputs with other ISoP members.

If you would like to get involved with any of the above projects, please do not hesitate to contact us with offers to help or with new ideas. Despite all the difficulties this year, we are confident that our professional society will remain committed to our mission of monitoring pharmaceutical products to improve patient safety worldwide.

\section{Compliance with Ethical Standards}

Funding No sources of funding were used to prepare this manuscript.

Conflicts of interest Rebecca Chandler, Deirdre McCarthy, JeanChristophe Delumeau and Mira Harrison-Woolrych have no conflicts of interest that are directly relevant to the content of this study.

Open Access This article is licensed under a Creative Commons Attribution-NonCommercial 4.0 International License, which permits any non-commercial use, sharing, adaptation, distribution and reproduction in any medium or format, as long as you give appropriate credit to the original author(s) and the source, provide a link to the Creative Commons licence, and indicate if changes were made. The images or other third party material in this article are included in the article's Creative Commons licence, unless indicated otherwise in a credit line to the material. If material is not included in the article's Creative Commons licence and your intended use is not permitted by statutory regulation or exceeds the permitted use, you will need to obtain permission directly from the copyright holder.To view a copy of this licence, visit http://creativecommons.org/licenses/by-nc/4.0/.

\section{References}

1. https://www.who.int/emergencies/diseases/novel-coronaviru s-2019/global-research-on-novel-coronavirus-2019-ncov/solid arity-clinical-trial-for-covid-19-treatments. Accessed 9 April 2020

2. Le Thanh T, Andreadakis Z, Kumar A, Gómez Román R, Tollefsen $\mathrm{S}$, et al. The COVID-19 vaccine development landscape. Nat Rev Drug Discov. 2020. https://doi.org/10.1038/d41573-02000073-5. 